\title{
Total avulsion of an osseointegrated screw
}

\author{
B. J. O'Reilly, C. Walter (Heiden, Switzerland)
}

\begin{abstract}
The close union achieved between pure grade titanium and healthy bone makes dislocation a rare event. We report the case of the total avulsion of an apparently well-integrated implant from a healthy mastoid bone.
\end{abstract}

\section{Introduction}

Since Professor Branemark first reported the phenomenon of osseointegration (Branemark et al., 1969) over 100,000 pure grade titanium screws have been implanted in the skull, mostly intraorally. A feature of these implants is their remarkably strong adhesion to the surrounding bone which makes rejection or dislocation an exceptional event.

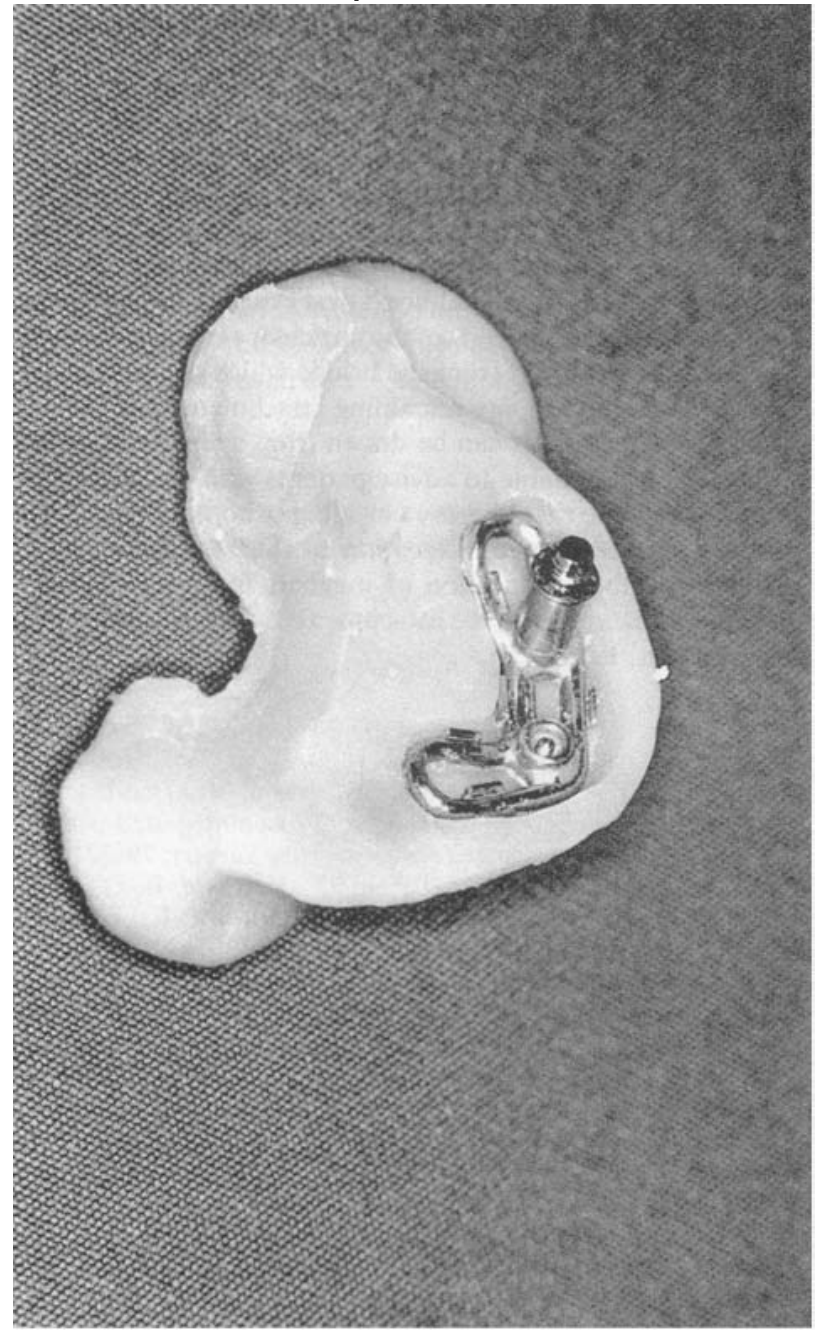

FIG. 1

Auricular prosthesis with superior fixture still attached.

\section{Case report}

The patient, a 17-year-old Caucasian and a semi-professional ice-hockey player, had a total agenesis of the right auricle. Seven years previously he had undergone an unsuccessful otoplasty and, when offered the choice of a revision otoplasty or a bone-anchored prosthetic auricle, opted for the latter.

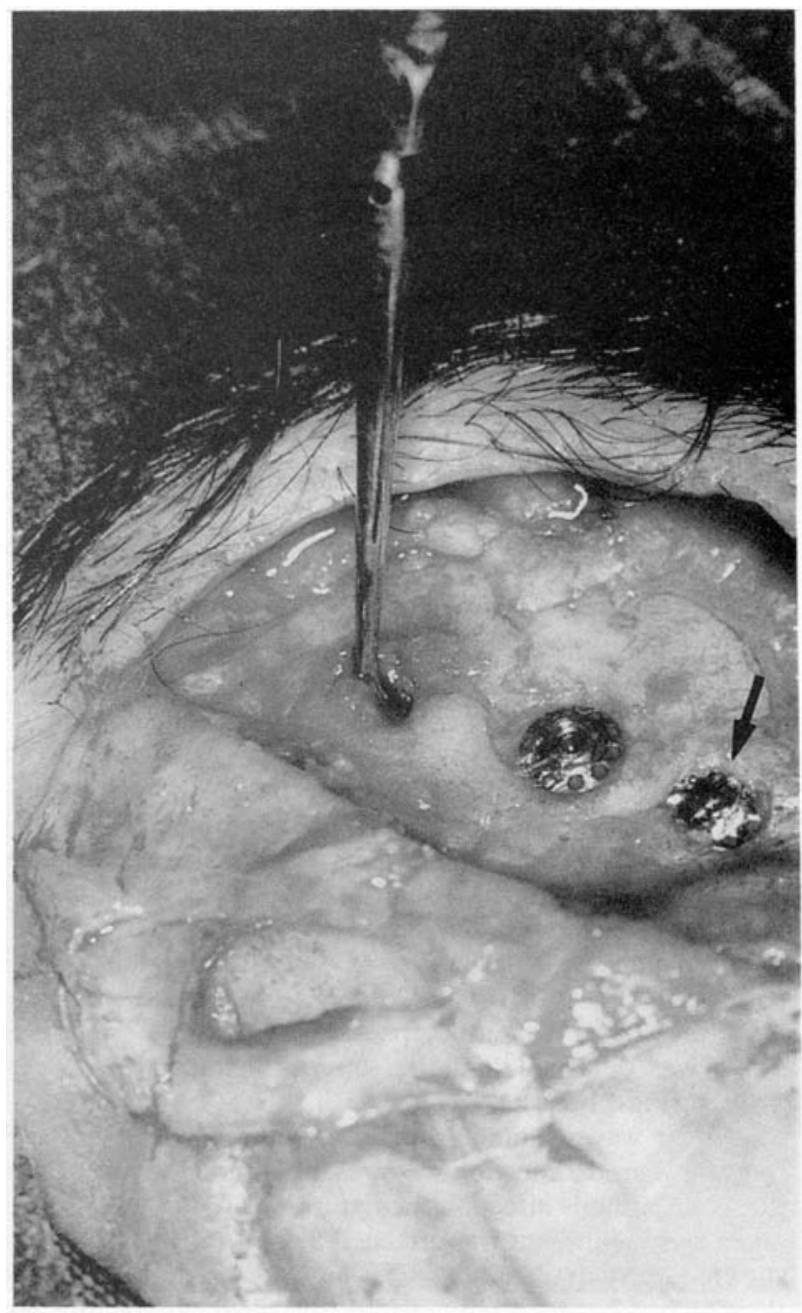

Fig. 2

Instrument indicating site of avulsed fixture. Fixture containing sheared abutment screw arrowed. 


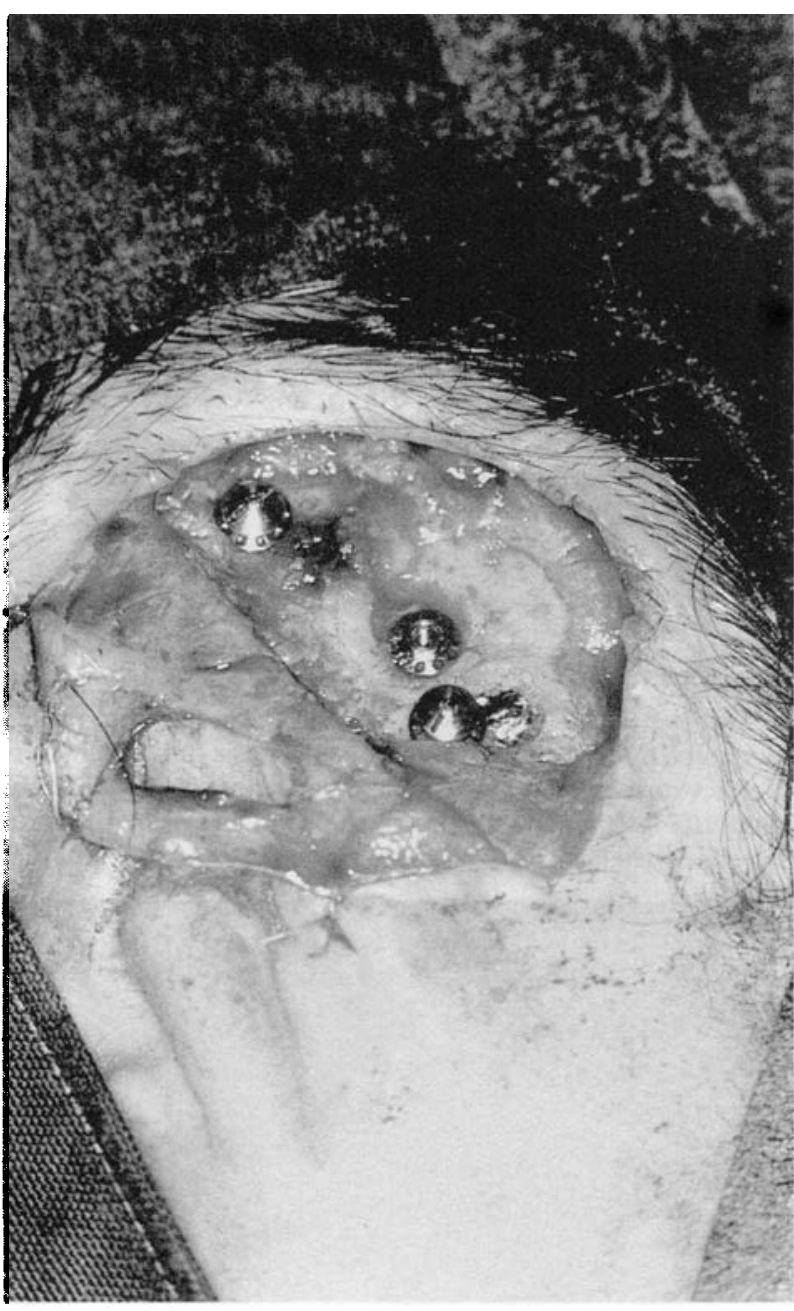

Fig. 3

Two replacement fixtures with cover screws.

The first stage surgery was performed in October 1989 and three $4 \mathrm{~mm}$ titanium screws were satisfactorily implanted by the senior author (CW) in the standard fashion (Tjellstrom, 1989) into healthy cortical mastoid bone. At the second stage surgery, performed four months later, all three fixtures were found to be rigid. Abutments were attached to the upper two fixtures without mishap but the abutment screw sheared within the lower fixture and could not be removed. Although useless, the lower fixture was left in situ and a bridge was attached across the two remaining abutments. A silicon rubber auricular prosthesis was fashioned around an acrylic plate with six retention clips to fasten it to the bridge.

The patient was delighted with his prosthesis and wore it daily until August 1990 when, during a game of ice hockey, he was struck by a club on the prosthesis. The inferior abutment screw was detached and the superior fixture was entirely avulsed (Figs. $1 \& 2$ ).

The ear was explored 10 days after the incident by which time a low grade infection was present around the inferior fixture. Despite this infection the fixture was found to be secure and a fresh abutment was attached to it. The superior fixture site contained no remaining part of its prosthesis and the screw threads had been entirely erased, although no fracture was present. Two new $4 \mathrm{~mm}$ titanium screws were implanted in fresh sites (Fig. 3) and the wound was closed with a split thickenss skin graft around the abutment.

We intend to attach a new bridge in four months and hope to persuade the patient to remove the new prosthesis before icehockey games.

\section{Discussion}

Although initially designed for intraoral use, osseointegrated implants have been used percutaneosly since 1977 to attach auricular, nasal and orbital prostheses and bone anchored hearing aids. The success (ie integration) rate appears to be at least as high for the percutaneous as for the intraoral implants. Albrektsson et al. (1987) reported the failure of only six out of 389 percutaneous implants, and five of these failures were in irradiated patients. Considering only auricular prostheses, the results are at least as good with only two failures from 282 implants (Tjellstrom, 1989). The one area of (relative) failure is the orbit where 19 out of 81 implants failed to integrate (Tjellstrom, 1989). However, the thin cortical bone available, the frequency of previous radiotherapy and the likelihood of penetrating the frontal sinus may all militate against success in this area.

Once osseointegration has occurred the union achieved between implant and bone is so close that its removal, whether by accident or design, is extremely difficult; a torque of $136 \mathrm{Ncm}$ has failed to remove a well-integrated implant (Tjellstrom, 1989). Consequently where it is necessary to remove a prosthesis it is usual to remove the abutment but to leave the osseointegrated screw as a permanent subcutaneous fixture. Equally it is not surprising that traumatic dislocation of an osseointegrated screw is a rare event. Of the prostheses secured by osseointegrated screws the auricle and the boneanchored hearing aid are perhaps the more exposed to trauma. The bone-anchored hearing aid readily detaches from its abutment and there is little likelihood of dislocating its fixture. In contrast, the retention clips of the auricular prosthesis may not be so readily detached from the bridge which (as in our case) may act as a lever on any remaining attachment.

No firm conclusions can be drawn from a single case but it would seem reasonable to advise patients with auricular prostheses to remove them before engaging in contact sports. The possibility of traumatic dislocation of the implant would be obviated by the substitution of magnets for retention clips. although these might make attachment of the prosthesis more awkward for the patient.

\section{References}

Albrektsson, T., Branemark, P. I., Jacobsson, M., Tjellstrom, A. (1986) Present clinical applications of osseointegrated percutaneous implants. Plastic and Reconstructive Surgery, 79: 721-730.

Branemark, P. I., Adell, R., Beine, U., Hansson, B. O. (1969) Intra-osseous anchorage of dental prostheses: 1. Experimental studies. Scandinavian Journal of Plastic and Reconstructive Surgery, 3: 81-100.

Tjellstrom, A. (1989) Osseointegrated systems and their applications in the head and neck. In Advances in Otolaryngology Head and Neck Surgery, (Myers, E. M., ed.), Year Book Medical Publishers Inc., 3: 39-70.

Address for correspondence:

Squ. Ldr. B. J. O'Reilly, F.R.C.S.Ed.

Princess Mary's R.A.F. Hospital,

Halton,

Aylesbury,

Bucks HP22 5PS

Key words: Ear, external; Osseointegrated implant 